Entre la intervención política y la construcción de un ethos profesional. La red de revistas estudiantiles independientes de medicina en la década del veinte Luciana Carreño

páginas / año 12 - n 30 Septiembre-Diciembre / ISSN 1851-992X/ 2020

http://revistapaginas.unr.edu.ar/index.php/RevPaginas

DOI: http://dx.doi.org/10.35305/rp.v12i30.442

\title{
Entre la intervención política y la construcción de un ethos profesional. La red de revistas estudiantiles independientes de medicina en la década del veinte ${ }^{1}$
}

\author{
In between political intervention and the construction of a professional \\ ethos. The network of independent medicine student's journals in the \\ twenties
}

\author{
Luciana Carreño \\ Consejo Nacional de Investigaciones Científicas y Técnicas, \\ Centro de Estudios en Historia, \\ Cultura y Memoria, Departamento de Ciencias Sociales, \\ Universidad Nacional de Quilmes (Argentina) \\ lcarreno1@uvq.edu.ar
}

\begin{abstract}
Resumen
El desarrollo de la Reforma Universitaria dio lugar a la emergencia de distintas revistas que actuaron como emprendimientos de intervención política cultural de los estudiantes identificados con ese movimiento. En el artículo se adoptó un abordaje sobre las revistas en el que se analizaron dichas prácticas de intervención intelectual de los estudiantes considerando también la vida universitaria en la cual se enmarcaban las publicaciones. Para ello se estudiaron los cambios sociales en la matrícula universitaria y los intereses profesionales de los alumnos como aspectos involucrados en la constitución de una red de revistas independientes de medicina que conectó a agrupaciones estudiantiles de las universidades nacionales de Buenos Aires y Córdoba, entre 1918 y 1926. Se sostiene que la red de publicaciones analizada expresó un nuevo tipo de periodismo estudiantil que surgió como consecuencia del proceso de democratización y organización que implicó la Reforma dentro del movimiento estudiantil. Asimismo, se afirma que este tipo de periodismo reformista estuvo singularizado por un especifico ethos estudiantil disciplinar y profesional, vinculado a la carrera de medicina, que no solo diferenció a estas publicaciones de las demás revistas del periodo, sino que también distinguió a las prácticas de intervención política-cultural del reformismo de medicina.
\end{abstract}

\section{Palabras clave}

\footnotetext{
${ }^{1}$ Este trabajo se enmarca en el proyecto posdoctoral "Sociabilidades juveniles en torno a la Reforma Universitaria y la experiencia democrática. Los modos de intervención pública y universitaria del asociacionismo estudiantil de la Universidad de Buenos Aires (1912-1930)" financiado por el Consejo Nacional de Investigaciones Científicas y Técnicas. Asimismo, forma parte del proyecto "Universitarios en Argentina. Proyectos científicos y prácticas académicas, culturales y políticas, entre el siglo XX y los inicios del XXI", financiado por el Departamento de Ciencias Sociales de la Universidad Nacional de Quilmes. Agradezco los comentarios realizados por los evaluadores anónimos de la revista Páginas.
}

Esta obra está sujeta a la Licencia Reconocimiento-NoComercial-Compartirlgual 4.0 Internacional de Creative Commons. http://creativecommons.org/licenses/by-nc-sa/4.0/ (cc) EY-NC-SA 


\title{
Luciana Carreño
}

Reforma Universitaria; Revistas; Medicina; Estudiantado; Profesión.

\begin{abstract}
The development of the University Reform gave way to the emergence of different reviews that acted as undertakings of political-cultural intervention for the students who identified with this movement. This article adopted an approach towards these reviews by which the student's practices of intellectual intervention were analyzed, while also considering the universitary life in which these reviews were framed. In order to achieve this, social changes in universitary enrollment and alumni's profesional interests were studied as aspects involved in the formation of an independent network of medicine school reviews that connected student organizations from Buenos Aires and Córdoba between 1918 and 1926. This research asserts that the network of publications that were analyzed expressed a new type of student's journalism that emerged as a consequence of the process of democratization and organization that the Universitary Reform entailed within the student movement. This study also affirms that this type of reformist journalism was differentiated by a specific student, disciplinary and professional ethos linked to medicine studies, which not only set apart these publications from others in this period, it distinguished the practices of political-cultural intervention in medicine school reformism.
\end{abstract}

\section{Keywords}

University Reform; Reviews; Medicine school; Student body; Profession.

\section{Introducción}

En paralelo al desarrollo del movimiento reformista, iniciado en 1918 por los estudiantes cordobeses, aparecieron una serie de revistas que se proponían renovar el periodismo y la vida estudiantil más allá de las cuestiones gremiales que predominaban en los centros y entidades organizativas estudiantiles. El movimiento de la Reforma no solamente impactaba en las estructuras académicas de las universidades del país, sino que también interpelaba un nuevo tipo de intervención intelectual por parte de los estudiantes. En las últimas décadas, diferentes autores han dado cuenta del papel de las revistas como emprendimientos de intervención política cultural del reformismo. Estos estudios contribuyeron, entre otros aspectos, a reconstruir y contextualizar la circulación de ideas de la Reforma (conocida hasta entonces a través de compendios documentales realizados por sus protagonistas) dentro de una trama más amplia de polémicas, proyectos editoriales y redes intelectuales de carácter trasnacional que posibilitaron a los estudiantes una activa participación en la vida pública. ${ }^{2}$

Desde otra perspectiva, el presente artículo propone un abordaje sobre las revistas en el que se analizan las prácticas de intervención político-cultural de los estudiantes considerando también la vida estudiantil en la cual se enmarcaban. Los cambios sociales en la institución y en la población universitaria; la conformación de ciertos habitus de trabajo intelectual que singularizaban las carreras

\footnotetext{
2 Dentro de esta línea, el análisis de las revistas se combina con el estudio de otras prácticas y modalidades de intervención política vinculadas al reformismo. Véase, entre otros (Bustelo, 2015; Graciano, 2008; Pita González, 2009; Rodríguez, 1999).
} 


\section{Entre la intervención política y la construcción de un ethos profesional. La red de revistas estudiantiles independientes de medicina en la década del veinte}

universitarias y la definición de un determinado ethos disciplinar- profesional en relación al perfil de los egresados, constituyen puntos de análisis desde los cuales se busca aportar en la compresión de las revistas y del estudiantado del periodo.

Para ello, en el artículo se toma como objeto de estudio un corpus de revistas estudiantiles que aparecieron en distintas universidades a partir del desarrollo del movimiento reformista. La vinculación de estas revistas (mediante canjes, saludos editoriales y la publicación compartida de artículos y colaboradores) permite la reconstrucción de una red y habilita el estudio comparativo de los puntos de análisis señalados en relación a las ciudades universitarias en las cuales estos proyectos editoriales se ubicaban. A partir de estas cuestiones, en el artículo se reconstruye una red conformada por las revistas La Cureta y El Cocobacilo (de la ciudad de Buenos Aires) y El Bisturí (de Córdoba).

La particularidad que define a estas publicaciones se relaciona con la especificidad disciplinar asociada a la carrera de medicina y por el abordaje de problemas propios de la vida estudiantil- universitaria. A su vez, estas revistas se distinguieron por el carácter independiente que adoptaron en relación a las publicaciones de los centros de estudiantes, por el cual se perfilaron como órganos de agrupaciones emergentes que buscaban renovar la vida asociativa estudiantil en sintonía con los postulados reformistas. A partir de estas cuestiones se sostiene que la red de publicaciones que reconstruimos expresó un nuevo tipo de periodismo estudiantil que surgió como consecuencia del proceso de democratización y organización que implicó la Reforma dentro del movimiento estudiantil. Asimismo, se afirma que este tipo de periodismo reformista estuvo singularizado por un específico ethos estudiantil disciplinar y profesional, vinculado a la carrera de medicina, que no solo diferenció a estas publicaciones de las demás revistas del periodo, sino que también distinguió a las prácticas de intervención política-cultural del reformismo de medicina. A partir de estas cuestiones, en el artículo se reconstruyen una serie de articulaciones, no exentas de tensiones y particularidades según los casos de estudio considerados dentro de la red, entre las formas de intervención política a la que alentaban estas publicaciones a través del reformismo y la construcción de un ethos profesional vinculado a la carrera de medicina.

La red de revistas independientes de medicina dentro de la trama del periodismo estudiantil

Los orígenes del periodismo estudiantil en el siglo XIX se ubican en estrecha relación con el desarrollo del movimiento asociativo en la segunda mitad de ese siglo, dentro del cual las revistas actuaron como órganos de prensa de las entidades culturales, profesionales y políticas vinculadas a la universidad. En el ámbito de la medicina, según ha estudiado González Leandri para el caso de Buenos Aires, la aparición de revistas se desarrolló en paralelo al surgimiento de sociedades médicas que buscaban comunicar específicas estrategias de legitimación frente a la elite de notables que gobernaba las instituciones universitarias, provinciales y estatales 


\section{Luciana Carreño}

relativas al "arte de curar" (1999a). Sin embargo, al igual que otras asociaciones culturales que también se desarrollaron de modo paralelo en el ámbito universitario porteño y cordobés, el perfil estudiantil de estas publicaciones resultaba aun poco definido tanto por la heterogeneidad de los redactores y temas abordados como por la convocatoria no limitada a un público universitario. ${ }^{3}$

En las primeras décadas del siglo XX, la definición de un perfil netamente estudiantil se concretó junto con la organización de los centros de estudiantes, los cuales, a diferencia del asociacionismo anterior, implicaron la creación de entidades específicas dentro de las distintas escuelas y facultades. En líneas generales, el tipo de publicación que acompañó la organización gremial de los estudiantes se caracterizó por la reproducción de apuntes correlativos a los programas de estudio, artículos de interés científico de las diferentes carreras y notas breves sobre distintos aspectos de la "vida estudiantil" o "universitaria" (turnos de exámenes, normativas, eventos sociales, etc.). ${ }^{4}$

El movimiento de la Reforma y, previamente, el impacto causado por la Primera Guerra Mundial y la Revolución rusa, implicaron la aparición de un nuevo tipo de periodismo claramente diferenciado del formato y de las cuestiones gremiales que predominaban en los centros. Tal como ha analizado Bustelo, a mediados de la segunda década, comenzaron a desarrollarse nuevas redes asociativas y editoriales, en las cuales surgió una identidad estudiantil en torno a distintas afinidades culturales y políticas. Según reconstruyó esta autora, esta transformación de la prensa universitaria no solo se expresó a través de una serie de revistas culturales que renovaban sus secciones, contenidos e intereses a tono con una nueva identidad estudiantil no referenciada en lo gremial, sino también en el surgimiento, a partir de 1919, de un periodismo político estudiantil que saludó el advenimiento de la Revolución rusa y que buscó convocar a un público de estudiantes y obreros (2015). En esa coyuntura la prensa de los centros también se transformó en paralelo a una mayor organización gremial que implicó la Reforma. Este proceso se materializó en la aparición de periódicos de las nuevas federaciones estudiantiles e implicó también nuevas secciones y contenidos en las revistas de los centros que comenzaron a reproducir distintos manifiestos y noticias del movimiento reformista a nivel latinoamericano. Sin embargo, la función de difusión científica continuó teniendo un peso gravitante en varias de estas publicaciones, en las cuales persistía la reproducción de artículos académicos relativos a las distintas carreras. En contraposición a esta tendencia, algunos centros comenzaron a publicar, de

\footnotetext{
3 Tanto para el ámbito cordobés como para el porteño en este periodo coexistieron destacadas revistas y asociaciones culturales cuyo reclutamiento era preminentemente universitario, junto con otros periódicos estudiantiles de vida efímera y que abarcaban una heterogeneidad de temas. Para el caso córdobas véase (Valiente, 2016) y para el porteño (Carreño, 2020).

4 Para 1916 compartían esas características las publicaciones de los centros de estudiantes por entonces existentes en las universidades de Buenos Aires, La Plata, Córdoba y Tucumán (Barbich, (jul. 1916). Los estudiantes y el adelanto intelectual del país. Crónica sobre las Revistas de los Centros de Estudiantes Universitarios de la República. Revista del Centro de Estudiantes de Ingeniería, XVII (169), pp. 139-176).
} 


\title{
Entre la intervención política y la construcción de un ethos profesional. La red de revistas estudiantiles independientes de medicina en la década del veinte
}

modo paralelo a las revistas, boletines de formato tabloide que recurrían a un tono satírico y abordaban solamente cuestiones propias de la vida universitaria y del movimiento estudiantil.

A partir de este sucinto recorrido sobre las características de la prensa universitaria, se puede establecer una primera caracterización de la red de revistas independientes de medicina. Al igual que las revistas culturales, estas publicaciones, de formato libro, reprodujeron en sus páginas notas y documentos que expresaban distintas prácticas de intervención política- cultural y formas de sociabilidad propias del reformismo (publicación de manifiestos; reproducción de cartas públicas; reseñas de viajes y mítines político- intelectuales, etc.). Sin embargo, a diferencia de estas últimas, se caracterizaron por el tono predominantemente humorístico y por el lugar que ocuparon en sus páginas las cuestiones relativas a la vida universitaria en temas también presentes en los boletines y revistas de los centros como los exámenes, el dictado de las clases o las formas de promoción dentro de la carrera académica. Asimismo, al igual que las revistas de los centros de medicina, retomaban aspectos presentes en la prensa asociativa médica en el abordaje de cuestiones centrales de la práctica profesional; el aparato publicitario especializado y la convocatoria de un mismo público universitario.

Pese a estas similitudes, tal como se advertía en la nota con la que la revista rosarina El Leucocito saludaba la aparición de su par cordobesa El Bisturí, el carácter satírico y/o critico tanto frente a la prensa gremial como a la netamente profesionalcorporativa constituyó un rasgo identitario sobre el cual se articuló la vinculación editorial entre las revistas independientes de medicina:

\begin{abstract}
Esta clase de publicaciones está llamada a tener éxito, como lo tiene "La Cureta" de Buenos Aires, como lo tuvo en otro tiempo "El Glóbulo Rojo" y como lo ha conseguido "El Leucocito" en todo el país y en el extranjero. Es una natural reacción contra las publicaciones científicas y pesadas que no logran ni siquiera herir la más pequeña fibra del espíritu, siendo inútil erudismo en competencia desventajosa con las bibliotecas. Saludamos muy cordialmente a "El Bisturí", quedando establecido el canje con "El Leucocito". 5
\end{abstract}

Esta reseña permite confirmar, desde una serie de rasgos editoriales a los que aludimos más arriba, la presencia de una red de revistas independientes de medicina, identificable por el uso de la nomenclatura médica al que recurrían para la denominación de las publicaciones. ${ }^{6}$ Junto con estas características genéricas,

\footnotetext{
${ }^{5}$ Agradecemos y retribuimos (jul. 1923). El Bisturí. I (4), p. 13.

${ }^{6}$ A partir de los nombres es posible identificar la difusión de este periodismo en distintas ciudades universitarias. Junto con las revistas citadas en Buenos Aires (El Glóbulo Rojo, La Cureta y EI Cocobacilo), Córdoba (El Bisturî) y Rosario (El Leucocito), se agrega el caso de la revista platense EI Forcep (1925), aunque difiere de las demás al recurrir al formato tabloide y no contener anuncios específicos de comunidad médica (fármacos, aparatos, etc.). Sobre esta publicación véase (Celentano, 2014).
} 


\section{Luciana Carreño}

entre las revistas estudiantiles La Cureta. Órgano de la agrupación de estudiantes de Medicina Pro Reforma (1918-1925); El Cocobacilo. Revista satírica mensual (19181924) y El Bisturí. Revista Estudiantil de Medicina (1923- 1926), resulta posible reconstruir una serie de vinculaciones concretas que conectaron al estudiantado porteño y cordobés representado en esas publicaciones.

La articulación de una red entre Buenos Aires y Córdoba se advierte explícitamente a través de un común elenco de colaboradores. El Cocobacilo, apareció en 1918 y tras un lapso volvió a editarse en 1920 hasta marzo de 1924. Fue fundada y dirigida por Jacobo Zimmermann, quien a su vez colaboraba en La Cureta con el seudónimo Obdulio Hierofonte (hijo). Posteriormente, la publicación quedó a cargo de José Svibel, conocido también como "Bel-Vis-Age". Por su parte, desde su aparición en 1918 La Cureta estuvo a cargo de José Belbey, presente también en la lista de colaboradores de El Cocobacilo junto con otros autores que publicaron en las dos revistas: Osvaldo Loudet, Julio Savón y Mario X. Landó.7 Paralelamente, La Cureta reseñó elogiosamente la aparición de $E 1$ Cocobacilo $^{8}$ y reprodujo un artículo de $E I$ Bisturí. ${ }^{9}$ Finalmente, esas mismas coincidencias se registran en relación a esa última revista, fundada en Córdoba por Abraham Schellemberg en 1923. En un saludo editorial, se expresaba la estrecha relación que unía a la revista cordobesa con El Cocobacilo, ${ }^{10}$ de la cual se reprodujeron varios artículos y caricaturas, a la vez que también incluyó colaboraciones de José Belbey.

En estas revistas, la reacción contra la prensa gremial- corporativa médica, que se advertía en El Leucocito, implicó como contrapartida construcción de un ethos juvenilista que combinaba al mismo tiempo una identidad estudiantil asociada a una serie de valores y prácticas de sociabilidad propias del reformismo (en su vertiente emparentada a posiciones políticas de la izquierda socialista) con la defensa y construcción de un determinado ideal profesional que retomaba y, a la vez pretendía reformular, antiguos preceptos ya asentados en la corporación médica.

\section{La construcción de una plataforma reformista en el estudiantado de medicina}

Los rasgos específicos que adoptaron estas publicaciones se expresaron también en la convocatoria a un público más acotado que el de otras revistas del periodo. Este tipo de interpelación, que apuntó principalmente al estudiantado de medicina, implicó la construcción de una plataforma reformista que se extendió en una doble dirección. Según se analiza en este apartado, dentro del movimiento estudiantil estas agrupaciones aspiraron a reformular las entidades gremiales en el contexto marcado por la irrupción del reformismo y por la posterior retracción institucional

\footnotetext{
7 Colaboradores de "El Cocobacilo" (feb.-marzo de 1924). El Cocobacilo, IV (53), pp. 8 -10.

8 "El Cocobacilo", (20/05/1919). La Cureta, II (6), p 30. Ese saludo correspondía a otro anterior publicado en el primer número de El Cocobacilo, lamentablemente no conservado.

${ }^{9}$ De El Bisturí (abril 1925). La Cureta, IV (16), p. 333.

10 De nosotros mismos (jun. 1923). El Bisturí, I (3), p. 11-12.
} 


\section{Entre la intervención política y la construcción de un ethos profesional. La red de revistas estudiantiles independientes de medicina en la década del veinte}

de este movimiento en la década del veinte. Por fuera de la universidad, estas revistas apostaron a la difusión de determinadas prácticas de intervención intelectual singularizadas por la matriz disciplinar y el perfil profesional de esa población universitaria.

En relación al primer punto, las vinculaciones de la red de revistas de medicina permiten contrastar una serie de características que diferenciaron la matrícula y la organización gremial estudiantil en la Universidad Nacional de Córdoba (UNC) y en la Universidad de Buenos Aires (UBA). La diferencia principal en este sentido deriva de la acusada concentración, en esta última, de la población universitaria a nivel nacional. Esta característica se advertía especialmente en la Facultad de Ciencias Médicas porteña (FCM), la cual para 1925 contaba con una inscripción de 4592 alumnos, que contrastaban con las cifras que respectivamente registraban las universidades del Litoral (1520), Córdoba (1327) y La Plata (448) (Ministerio de Justicia e Instrucción Pública, 1926).

Sin embargo, para el caso de medicina, más allá del factor poblacional, las diferencias en la organización gremial entre el estudiantado porteño y cordobés se vinculan con los distintos procesos de reconocimiento institucional de ambas facultades dentro del sistema universitario ${ }^{11} \mathrm{y}$, tal como profundizaremos más adelante, por el peso que jugaron en la UBA las asociaciones médicas de estudiantes y graduados en el proceso de profesionalización de dichas carreras. En el caso de Buenos Aires, la organización del Centro de Estudiantes de Medicina (CEM), fundado en 1900, se consolidó tempranamente mediante la fusión, en 1909, con el Círculo Médico Argentino (CMA). Gracias a esa unión, el CMA-CEM pasó a adquirir la personería jurídica y heredar una serie de atributos materiales y simbólicos de una de las principales asociaciones que, en el siglo anterior, participaron en la construcción del prestigio y la legitimación de la profesión médica. ${ }^{12}$ A partir de esto, en la década siguiente el CMA-CEM logró destacarse no solo como una de las principales entidades gremiales del estudiantado porteño, sino también por ser la primera en impulsar una serie de reformas en sus bases estatutarias que implicaron una democratización de sus mecanismos electorales en correspondencia con la reforma que se efectuaba a nivel nacional a través de la ley del sufragio universal masculino, secreto y obligatorio, sancionada en 1912.

Esa temprana democratización de las estructuras gremiales en el centro de medicina posibilitó la aparición de nuevos liderazgos estudiantiles, cuyo prestigio no dependía necesariamente como antes del capital social y político que se movilizaba

\footnotetext{
${ }^{11}$ A diferencia de la UBA, cuya FCM se instituyó con la creación de la universidad en 1821, en la UNC la creación de la Facultad de Medicina se dio en 1877. En 1891 su escasa matriculación fue uno de los argumentos que se esgrimieron desde un proyecto de la Comisión de Presupuesto del Congreso Nacional, a favor de cerrar dicha Facultad, lo cual finalmente no se llevó a cabo (Agüero, 2017: 97). 12 Gracias a la fusión, el CEM obtuvo su propio local y heredó la biblioteca del CMA. Esta condición le aportó una importancia adicional ya que funcionaba al mismo tiempo como lugar de sesión de la Federación Universitaria de Buenos Aires y de las actividades de otros centros que eventualmente lo solicitaban.
} 


\section{Luciana Carreño}

en época de elecciones, sino que también podía legitimarse dentro del variado arco del asociacionismo juvenil (en la participación cotidiana en distintas agrupaciones o través de la oratoria en distintos actos públicos) que se desarrolló en el contexto de la Gran Guerra, la Revolución rusa y la Reforma. A partir de esa coyuntura, el proceso de democratización de los centros de estudiantes porteños adquirió mayor complejidad a partir de las diferencias políticas e ideológicas que posicionaron de distinto modo al alumnado sobre el programa que debía caracterizar al movimiento reformista. La consecuencia de ese proceso implicó la aparición de un nuevo tipo de asociacionismo dentro de la vida universitaria, que se manifestó a través de la multiplicación de agrupaciones que comenzaron a disputar la conducción gremial e inclusive a competir de modo paralelo con las funciones de los centros (Carreño, 2020).

La aparición de La Cureta y EI Cocobacilo se enmarcan en ese proceso de democratización que se profundizó con la Reforma. La trayectoria de José Belbey, director de La Cureta, es representativa de los nuevos liderazgos estudiantiles que comenzaron a construirse a través de la experiencia asociativa extrauniversitaria y la militancia que movilizó la experiencia reformista en la ciudad. Este estudiante, que llegaría a destacarse como un activo intelectual del Partido Socialista (Tarcus, 2007: 55), fue integrante del Ateneo Universitario, asociación que desde su creación en 1914 se caracterizó por promover instancias de asociación con afanes culturales y por su enfrentamiento a los males de la universidad (Biagini, 2012: 199). Posteriormente, al iniciarse el movimiento reformista, participó de los actos callejeros que promovió la Federación de Asociaciones Culturales, agrupación fundada por Gregorio Bermman en apoyo a los estudiantes cordobeses que, entre 1918 y 1919, desplegó una serie de actividades con la adhesión de asociaciones de inscripción socialista (Bustelo, 2015: 185-193). Junto a estas actividades, su labor frente a La Cureta, le permitió destacarse en el ambiente estudiantil de medicina y ocupar la presidencia del CMA-CEM entre 1920-1921. El programa que acompañó a esa gestión en la conducción del centro y que animó la redacción de la revista, buscó alentar la reformulación de la entidad gremial mediante la extensión de sus funciones por fuera del ámbito universitario. Para ello, a tono con la propuesta de Federación de Asociaciones Culturales, desde la revista se apeló a seguir el ejemplo de la juventud cordobesa que: "se estrecha en fraternal abrazo con el proletariado, se une a él convencida que su alma forma parte integrante del pueblo que sufre y que trabaja y rompiendo con los convencionalismos anticuados, con las tradiciones ridículas no vacila un solo instante en marchar en pos de la bandera roja...". ${ }^{13}$ Por su parte, si bien en El Cocobacilo no se registran este tipo de posicionamientos políticos de izquierda, esta revista saludó los emprendimientos editoriales de esa filiación como Bases, Clarín, representantes del periodismo político afín al Partido Socialista Internacional, y a las cordobesas Mente y La Gaceta universitaria (Bustelo \& Domínguez, 2017). Asimismo, al igual que La Cureta, actuó como plataforma para

\footnotetext{
13 Vargas, H., Ejemplo de la juventud Cordobesa (25/09/1918). La Cureta I (5), p. 117.
} 


\section{Entre la intervención política y la construcción de un ethos profesional. La red de revistas estudiantiles independientes de medicina en la década del veinte}

promoción de fórmulas electorales que pretendían ampliar las funciones del centro por fuera de los asuntos meramente gremiales que solían ocupar un lugar predominante en la asociación. En 1923, El Cocobacilo auspició la fórmula DezeoBarrera, cuya propuesta tenía entre sus puntos centrales el afianzamiento de la Reforma Universitaria y en lo que respecta a las funciones del centro: la "extensión universitaria efectiva; Labor intensiva del Ateneo. Biblioteca Cultural y creación de un campo de deportes". 14

A diferencia del centro estudiantil porteño, la organización del estudiado cordobés de medicina no solo se institucionalizó posteriormente, ${ }^{15}$ sino que también resultó profundamente alterada tras la intervención de esa casa de estudios en 1923, decretada por el Poder Ejecutivo Nacional. Esta situación repercutió en la disolución de la Federación Universitaria de Córdoba (FUC) y del Centro de Estudiantes de Medicina, tras una fallida huelga estudiantil en contra de la medida del gobierno nacional (Schenone, 2009). En ese contexto, si bien El Bisturí, en contraste con las revistas porteñas, no se constituyó como un órgano de prensa de una determinada agrupación estudiantil que luchaba por la conducción gremial, representaba las aspiraciones de una fracción del estudiantado de medicina emparentado con los principales líderes que impulsaron el movimiento reformista en 1918. ${ }^{16}$ Desde esas páginas, la revista impulsó la reorganización de las entidades estudiantiles en oposición directa con otras agrupaciones que había acordado con las autoridades de la intervención asumir la representación del alumnado. ${ }^{17}$ Para ello El Bisturí actuó como hoja de difusión de los comunicados de la nueva FUC que volvió a constituirse a partir de 1924, en reclamo de un nuevo estatuto y una nueva finalidad "eminentemente social" para la universidad, la cual debía posicionarse entre el patrón y el obrero en defensa de la "justicia que anima los grandes movimientos de emancipación económica, política y social que anida en el espíritu de la nueva generación". ${ }^{18}$ Pese a esta función de propaganda la revista no evitó manifestar ciertos recelos frente la circunstancial organización de las entidades estudiantiles:

...los propósitos de un Centro de Estudiantes no deben ser tan solo de combate y de crítica, es indispensable que desarrolle una labor metódica entre el estudiantado y la población en general, inculcando nociones de índole diversa entre esta última, en forma de extensión universitaria, y haciendo conciencia progresista en el primero.

\footnotetext{
${ }^{14}$ Manifiesto (feb. 1923). El Cocobacilo, V (41) p. 11.

${ }^{15}$ La fundación del Centro de Estudiantes de Medicina de la UNC, se llevó a cabo en 1909. Sin embargo, antes de esa fecha se registran también asociaciones estudiantiles y profesionales de medicina, de poca duración (Garzón Maceda, 1916: 537-546).

16 Las vinculaciones con líderes del 18 (como Gregorio Bermann, Enrique Barros, Gumersindo Sayago, Jorge Orgaz) se registran en la publicación de documentos, discursos y en el seguimiento de sus trayectorias profesionales y docentes.

17 Galeno. Por fin "Ellos" son centro de medicina (jul. 1923). El Bisturí, I (4), p. 7.

${ }^{18}$ Manifiesto de la F.U. de Córdoba. A la nueva Generación y a los espíritus libres de América. (en. 1924). El Bisturí, III (32), p. 14.
} 


\section{Luciana Carreño}

Una biblioteca, una publicación, conferencias científicas, consultorios gratuitos, etc. es obra normal de un Centro de Estudiantes.

Buenos Aires puede darnos un ejemplo magnífico y sorprendente. Las agrupaciones estudiantiles allí existentes viven una lozanía siempre renovada. Sus movimientos de lucha contra la reacción universitaria no constituyen un obstáculo para la realización de una actividad digna de jóvenes universitarios. ${ }^{19}$

A través de estas referencias mutuas, en la cuales las juventudes cordobesas aparecían destacadas en La Cureta por su compromiso social y las porteñas, en el comentario de El Bisturí, por su grado de organización institucional, se observa otro de los puntos de vinculación entre estas revistas. Las tres publicaciones compartieron una misma función de crítica hacia las costumbres del estudiantado como principal obstáculo para la realización del programa reformista dentro y fuera de la universidad. Estas críticas identificaban negativamente distintas tipologías estudiantiles (los "olfas", los "niños bien" o los "patoteros"), cuyas conductas (obsecuencia, oportunismo, frivolidad, desinterés por los asuntos universitarios y sociales, xenofobia y violencia, entre otras) ${ }^{20}$ no cuadraban con el perfil estudiantil que los reformistas deseaban sociabilizar. Este perfil no solo se caracterizaba por el compromiso en la renovación educativa que promovía la Reforma sino también por la denuncia de las injusticias que sufrían los sectores sociales menos favorecidos.

Asimismo, este tipo de críticas, que recalaban en determinadas características, prácticas y consumos del estudiantado (la ostentación en el vestir, los apellidos ilustres, la frecuencia a determinados lugares distintivos de recreación) dan cuenta de una trama material y de ciertos habitus en los cuales se constituían las diferencias dentro de la universidad. De este modo, estas revistas expresan un proceso de diversificación de la matrícula universitaria que se operó en las primeras décadas del siglo, mediante el ingreso de estudiantes que representaban, en sus trayectorias familiares, a una primera generación que accedía a los estudios superiores. ${ }^{21} \mathrm{El}$ caso de Abraham Schellember director de El Bisturí, estudiante judío procedente del entonces imperio ruso, se ubica en correspondencia con el considerable porcentaje de estudiantes de origen extranjero que encontramos en la UBA, ${ }^{22}$ y que, como se ve en el caso de Schellemberg, se extendía también en la UNC. Al realizar sus estudios secundarios en Buenos Aires, antes de trasladase a Córdoba, este estudiante se conectó "estrechamente con compañeros hijos de la "colectividad" (Schellemberg,

\footnotetext{
${ }^{19}$ ¿Y el Centro? (ab. 1925) El Bisturí. II (25), p. 11.

20 Las actitudes violentas de los "niños bien" se referían a la participación de los estudiantes en la represión obrera, durante la "Semana Trágica", a partir de la huelga del taller Vasena en enero de 1919. En El Cocobacilo la crítica a los "niños bien" recogía la referencia literaria de Arturo Canela, autor del El Cocobacilo de Herrlin (obra que inspiró el nombre de la revista) y de Una Semana de Holgorio (diario de un Guardia Blanco), publicada en febrero de 1919. En esa obra Cancela retrataba por medio de la sátira, los episodios de la Semana Trágica, a través de los ojos de un niño bien. Estas mismas referencias y colaboraciones de Cancela también se observan en El Bísturí.

21 Ese proceso de diversificación también se efectuó con el ingreso de las mujeres a la educación superior, el cual comenzó a finales del siglo XIX en el campo de la medicina (Lorenzo, 2016).

22 Para 1920, el $14 \%$ de los estudiantes inscriptos de medicina había nacido en el extranjero. Dentro de ese porcentaje se observa una mayoría de estudiantes rusos y de Europa del este, de origen judío, los cuales habían ingresado al país con sus familias en la primera década del siglo (Carreño, 2020).
} 


\section{Entre la intervención política y la construcción de un ethos profesional. La red de revistas estudiantiles independientes de medicina en la década del veinte}

1982) y con los "centros socialistas", atraído "no sólo por las conferencias, sino por las lecturas que ofrecían sus bibliotecas". ${ }^{23}$ En estos contactos es posible advertir otro de los puntos que contribuyó a vincular la red de revistas de medicina. Por una parte, en las trayectorias de Belbey y Schellember, se advierte una vinculación compartida dentro de las formas de sociabilidad letrada en centros obreros y socialistas. Por otra parte, tanto El Bisturí como El Cocobacilo, contaron con directores que procedían de familias de inmigrantes judíos, a la vez que en La Cureta también se identifican colaboradores de ese origen. Asimismo, las dos primeras promocionaron activamente la revista Vida Nuestra (1917- 1923) una lograda publicación sobre cultura judía que contó la participación de Jacobo Zimmermann, director de El Cocobacilo. Este componente inmigratorio se expresaba también en las tres revistas en el repudio a la "xenofobia universitaria", que se operaba en los concursos de la $\mathrm{UNC}^{24}$ y en los de la asistencia municipal porteña mediante el requisito de "ser argentino" 25 o en las "inscripciones insultantes" contra un estudiante de origen judío en el internado médico que cursaban los estudiantes de la UBA. ${ }^{26}$

Asimismo, la diversificación que experimentaba la matrícula universitaria, en paralelo a una cierta ampliación de la oferta escolar en el nivel secundario, se observa en las tres publicaciones en las alusiones a los costos materiales de los estudios (aunque ello no se tradujo en una argumentación a favor de la gratuidad de la universidad) y en la participación en la redacción de varios "estudiantes pobres", alumnos que debían a recurrir a exoneraciones de derechos arancelarios para poder realizar sus estudios. ${ }^{27}$ La importancia de los costos materiales figuró como uno de los factores que contribuyeron a la edición de El Bisturí. En las memorias de su director se señalaba que el propósito de crear una revista coincidió con la posibilidad, que tenía su amigo y posterior colaborador de la revista Jacobo Goodbar, de "conseguir publicidades de productos médicos, farmacias y laboratorios por sus relaciones con las droguerías de Buenos Aires”, lo cual permitió no solo lograr una regularidad en la aparición de la revista sino también "cumplir con [las] escasas necesidades económicas" que tenía su director durante sus estudios (1982). Este rasgo, que implicó una marcada presencia de publicidades del rubro médico- farmacológico, fue compartido por El Cocobaciloy, en menor medida, por La Cureta.

${ }^{23}$ Los 101 años de Abraham Schellemberg. Eterna y fértil juventud [Entrevista] (27/10/1997). Clarín, Buenos Aires. La militancia adolescente en el socialismo que recordaba Schellember en esa entrevista, no figura en sus memorias publicadas en 1982. En ese libro destacaba también su amistad, a finales de la década del veinte, con Ramón Cárcano, gobernador de la provincia entre 1925 y 1928. ${ }^{24}$ Xenofobia universitaria (mayo 1924). El Bisturí, II (14), pp. 7-8.

25 Viale, A. Artículo 1으: Ser argentino (25/8/1918). La Cureta, I (3) pp. 53- 54.

${ }^{26}$ E.O. Carta Abierta (marzo 1920). El Cocobacilo, II (8), p. 1.

27 La denominación institucional de "estudiantes pobres" incluía la certificación de carencia de recursos como requisito para tramitar la exención. Sin embargo, ello no permite reducir estos pedidos a variables socioeconómicas, dado que los mismos respondían a criterios más complejos, entre los que se cuentan las concepciones de pobreza propios de esa época (Carreño, 2020). 


\section{Luciana Carreño}

Finalmente, el programa reformista que desplegaron estas revistas no solo se expresó las disputas por la conducción y organización de las entidades gremiales y en la "función de hacer conciencia en la masa estudiantil" 28 sino también mediante la promoción de ciertas prácticas de intervención intelectual que buscaban orientar la producción de saber académico en vinculación con las problemáticas sociales y políticas. Para ello los estudiantes contaban con el modelo que proveía la extensión universitaria, promovido en las tres revistas, como práctica adoptada por el reformismo que buscaba divulgar los saberes específicos de cada disciplina universitaria a través de distintos formatos de conferencias. Tal como mencionamos, El Cocobacilo auspició la fórmula Dezeo- Barrera, la cual incluía como primer punto de su agenda reformista la realización efectiva de la extensión, punto que aludía a la escasa participación del alumnado en esas actividades. Una vez a cargo de la presidencia del centro, en el periodo 1923-1924, esa comisión logró destacarse por el número de cursos de extensión, para lo cual se optó por el formato de las conferencias populares en locales obreros. ${ }^{29}$ Por su parte, desde El Bisturíse alentaron otras modalidades, para lo cual se instaba a los estudiantes y profesores a promover campañas de profilaxis social por medio de conferencias radiotelefónicas ${ }^{30}$ y se publicaron las conferencias de extensión a cargo de Gregorio Bermann, dirigidas a un público universitario y desplegadas de modo institucional mediante un convenio entre la UBA y la UNC. ${ }^{31}$

Por otra parte, se registra la promoción de otras iniciativas de intervención pública, vinculadas dentro de una trama de asociaciones médicas, proyectos institucionales universitarios y dispositivos de la salud pública, que vehiculizaban medidas provenientes del movimiento higienista y del entonces novedoso movimiento eugenésico. Estas alusiones figuraron en la adhesión a las campañas de la Liga contra la Tuberculosis; en la reseña sobre la constitución de la Asociación Eugénica Nacional; en los homenajes que se tributaban a destacadas figura del movimiento higienista $^{32}$ o en la institucionalización en la FCM de la UBA de la extensión universitaria desempeñada de forma conjunta entre el CMA-CEM y la cátedra y el Instituto de Higiene. A partir de esto, puede observarse que las modalidades de intervención pública que promovían estas revistas resultaban en parte tributarias

\footnotetext{
${ }^{28}$ Nuestro aniversario (mar. 1924) El Bisturí, II (12), p. 4.

29 Distintos testimonios señalaban la escasa participación de los socios en esas actividades. Por el contrario, la comisión Dezeo-Barrera fue citada posteriormente por el modo ejemplar en la realización de la extensión, en el dictado de veinte conferencias que contrastaban con las seis del periodo posterior (Fiori, V. (mayo. 1926). Memoria correspondiente al periodo 1925- 1926. Revista del CMA-CEM, Tomo XXVI (296), pp. 326-386).

30 Estación radiofónica de la casa Lutz, Ferrando y Cía. Campaña de Profilaxis (feb. De 1924). EI Bisturí, II (11), p. 8.

${ }^{31}$ Etiopatogenia de las toxicomanías por el Dr. Gregorio Bermann (nov. 1925). El Bisturí, III (31), p. 6.

32 En El Bisturí figuran homenajes a destacados higienistas como Rawson (Rawson (jun. 1924). EI Bisturí, II (15), p. 4.) y Súnico (Dr. Francisco P. Súnico (mayo 1925). El Bisturí, II (26), p. 6). A su vez, en el último año de El Cocobacilo se caracterizó por reproducir artículos de Eduardo Wilde y José Ramos Mejía, a la vez que incluyó colaboraciones de profesores, como Gregorio Araoz Alfaro, responsable del Departamento Nacional de Higiene.
} 


\section{Entre la intervención política y la construcción de un ethos profesional. La red de revistas estudiantiles independientes de medicina en la década del veinte}

de los preceptos que, de la mano del movimiento higienista, legitimaban la profesión médica a través de la figura del médico social, que combinaba los saberes específicos de la ciencia con una vocación social basada en ideales filantrópicos y humanitarios (Vezzetti, 1985: 36-42 ; Di Liscia, 2002: 262-264). Sin embargo, estas referencias aparecían reformuladas desde posiciones críticas que cuestionaban los márgenes de intervención social- profesional de los médicos dentro de las injusticias imperantes dentro del régimen social. Así, desde El Cocobacilo se adhería a la campaña de recaudación de fondos que promovía la Liga Argentina contra la Tuberculosis en el Día de la Flor. Sin embargo, luego de resaltar los valores nobles y desinteresados en la obra de su director, el profesor Gregorio Aráoz Alfaro, advertía sobre:

"el error en que muchos caen, cuando piensan que la caridad es capaz de resolver las cuestiones sociales. Apenas si es un paliativo (...) En buena ley, había que ir bien hondo para desarraigar las causas productoras y subsanarlas a objeto que, en lo sucesivo, no haya más día de la flor, sino días de una sociedad más armónica y perfecta capaz de desarrollarse sin dejar atrás de sus pasos las víctimas de su propio modo de ser". ${ }^{33}$

A su vez desde La Cureta, Lázaro Sirlin saludaba la creación de la Sociedad Eugénica Nacional pero, a diferencia de sus fundadores y otros profesionales dentro del campo médico (Vallejo, 2018), no recomendaba orientar su acción hacia "medidas prohibitivas y coercitivas" "propuestas en otros países", sino combatir los factores inmediatos de la degradación de la especie humana (la tuberculosis, el alcoholismo y la sífilis) y su causa profunda, identificada en "la miseria y todo lo que de ella depende (la alimentación, exceso de trabajo, etc), mediante una "sabia legislación social". 34

Por su parte, en El Bisturí las críticas se orientaron contra la realización de los Congresos Universitarios, organizados anualmente desde 1923 en distintas universidades nacionales bajo la iniciativa del rector de la Universidad Nacional de La Plata, Benito Nazar Anchorena. Al constituirse como estrategias de intervención intelectual colectiva en un variado repertorio de asuntos públicos, la realización de estos congresos coincidía con el propósito de la revista al promover una función social para la universidad opuesta a la inercia profesionalista que predominaba en las unidades académicas (Graciano, 2016). Pese a esta coincidencia, El Bisturí adhirió a la circular de la FUC, que convocaba a las entidades a no participar en el congreso a celebrarse en la UNC en 1925,35 e ironizó sobre los resultados y el alcance

\footnotetext{
33 El día de la Flor (sep. 1920). El Cocobacilo, II (14), p. 16.

34 Sirlin, L. La Sociedad Eugénica Nacional (25/08/1918). La Cureta, I (3), pp. 56-58.

35 La circular de la FUC argumentaba que el congreso era convocado por autoridades afines a la intervención y que no ofrecía "ningún interés científico, adquiriendo solo los contornos de una mera reunión burguesa" (El Congreso Universitario y los Estudiantes de Córdoba (sep. 1925). El Bisturí, III (30), p.4).
} 


\section{Luciana Carreño}

social de esas reuniones científicas que en total "duraron cinco horas, (...) ocho banquetes y cuatro bailes en los salones más aristocráticos de nuestra ciudad". ${ }^{36}$ Finalmente, otra modalidad de proyección socio cultural a la que apostaron estas publicaciones se orientó a la reformulación del carácter profesionalista de la universidad, a través de una apuesta de renovación científica y social de su perfil académico. Ello se expresaba en La Cureta, en la propuesta de renovación de los planes de estudio mediante la creación de una cátedra de medicina social, ${ }^{37}$ mientras que, en el ámbito cordobés, El Bisturí apoyó el programa auspiciado por Gregorio Bermann y Enrique Barros a favor de la creación de institutos y de reformulación de los planes de estudio a través de la contratación de un conjunto de "reputados sabios" contactados por el mismo Barros durante su estancia en Alemania. ${ }^{38}$ Sin embargo, tal como se denunciaba en repetidas ocasiones en la revista, los alcances de programa de renovación institucional quedaron limitados por los efectos de la intervención de la universidad, decretada en 1923.

De este modo, la difusión del modelo de universidad científica y social, actuó como otro de los puntos que vincularon a estas publicaciones y que a la vez las relacionaba con otras agrupaciones dentro de una red editorial más densa y variada que convocaba al público universitario reformista. ${ }^{39}$ Sin embargo, de modo específico, en paralelo a la formulación de ese ideal de renovación científica, las tres publicaciones se orientaron a la discusión de un nuevo ideal profesional que integrara los postulados reformistas con nuevos desafíos que se planteaban en el campo de acción de la profesión médica.

\section{La construcción estudiantil de un ideal profesional: mecanismos de reclutamiento y el ejercicio de la medicina}

Tal como señalamos anteriormente, desde el siglo XIX, la publicación de revistas figuró como una pieza central dentro de las estrategias de determinadas agrupaciones de estudiantes y graduados en el proceso de profesionalización de la medicina. En este aspecto, es posible advertir los vínculos entre la profesionalización, como proceso estructurante de estratificación de la nueva sociedad del siglo XX, y los cambios dentro del sistema educativo por los cuales se

\footnotetext{
${ }^{36}$ El Congreso Universitario. Sus resultados (nov. 1925). El Bisturí, III (31), p. 4.

${ }^{37}$ Rubinstein, A. Medicina Social (25/07/1918), La Cureta, I (1), pp. 6-8.

${ }^{38}$ Barros, E. La trisecular de las campanillas [discurso] (ag. 1924). El Bisturí, II (17), pp. 4-6.

${ }^{39}$ En el modelo propuesto para la universidad, las tres revistas se referenciaron en la obra y la figura de José Ingenieros. Se citan sus conferencias del Ateneo del CMA- CEM durante la presidencia de Belbey, sobre "La universidad del porvenir" (Ingenieros, 1920), en la cual se señalaba la función de las universidades de "fijar principios, direcciones, ideales que permitan organizar la cultura superior en servicio de la sociedad" (1922: 2). Según Bustelo, la acción reformista de Gregorio Bermann fue el enlace entre los reformistas cordobeses e Ingenieros, quien fue clave en la construcción de una identidad política reformista ligada al latinoamericanismo antiimperialista (2015: 197). El Bisturí participó en la construcción de esa identidad, mediante la publicación de noticias y documentos sobre el movimiento estudiantil latinoamericano y sobre destacados líderes antimperialistas como el peruano Haya de la Torre y el cubano Mella.
} 


\section{Entre la intervención política y la construcción de un ethos profesional. La red de revistas estudiantiles independientes de medicina en la década del veinte}

comenzaron a introducir distintos requisitos de incorporación que fueron definiendo las características de los grupos académicos y profesionales, según sus propios objetivos sociales, culturales y económicos. Asimismo, tanto la implementación de esos requisitos como las demandas de renovación del sistema educativo se orientaban a otorgar respetabilidad y prestigio social a los nuevos profesionales cuya legitimación pasaba a basarse en las calificaciones y los reconocimientos obtenidos en el campo universitario (González Leandri, 1999b). Junto con los cambios en el sistema académico, la historia de la legitimación social de las profesiones está ligada al reconocimiento y las regulaciones estatales, las cuales contribuyen de modo determinante, junto con la inserción de los especialistas profesionales dentro de las burocracias técnicas estatales, a la consolidación de los procesos de profesionalización en sus distintas modalidades. ${ }^{40}$

En el ámbito universitario porteño, la aparición de estas revistas, que disputaban los espacios de poder que ostentaba la elite médica al frente de la universidad, contribuyeron a socializar distintos preceptos y prácticas profesionales para asegurar mecanismos de reclutamiento y consolidar el prestigio de la profesión dentro y fuera de la comunidad médica. Se explica de este modo la defensa de los concursos; la promoción de los estudios libres y, de la mano del movimiento higienista, la proyección de la figura de médico como árbitro social, dentro las principales revistas del periodo, los Anales del Círculo Médico Argentino y la Revista Médico Quirúrgica (González Leandri, 1999a).

En la UNC, pese a la posterior definición institucional de la Facultad de Medicina (FM), el proceso de profesionalización de los médicos cordobeses también se desarrolló activamente en relación al movimiento asociativo médico. En 1894 se constituyó la Asociación de Médicos y Alumnos, agrupación que puede ser considerada como el antecedente directo de la creación, en 1910, del Círculo Médico de Córdoba (Rivero, 2019). Sin embargo, a diferencia del papel impugnador que desarrollaron las asociaciones porteñas en relación a la universidad, en el ámbito cordobés la FM y el Círculo Médico con su Revista, funcionaron conjuntamente (Rodríguez, 2007).

Otro punto de contraste, derivado de la función crítica que asumió el asociacionismo universitario-profesional en Buenos Aires, se relaciona con el papel que desempeñaron las asociaciones estudiantiles en promover la reforma de los estatutos de la UBA en 1906. Si bien, en sus causas inmediatas, los desencadenantes que motivaron este movimiento se relacionaban con aspectos puntuales que

\footnotetext{
40 La intervención del Estado, como factor político en los procesos de profesionalización, ha sido señalada para los casos de la Europa continental (en los que el factor estatal resultó determinante) e inclusive para los angloamericanos típicos del modelo de laizze -faire (donde la obtención del monopolio del saber para una profesión recae principalmente en la acción de los mismos profesionales) (Sarfatti Larson, 1989). En el caso argentino, este factor se destacó de modo relevante para todo el campo profesional (Frederic, Graciano \& Soprano, 2010) y de modo especial para el caso de la medicina (Gutiérrez Leandri, 1999a).
} 


\section{Luciana Carreño}

remitían a las estrictas condiciones de exámenes y los costos de los derechos universitarios, otros reclamos cuestionaban la legitimidad científica de las autoridades académicas y demandaban la mejora del profesorado a través de la docencia libre y la implementación de nuevos mecanismos en su reclutamiento (Bargero, 2002). A partir de esa reforma, la universidad porteña avanzaba, aunque con muchas limitaciones, en la creación de premios a la investigación y de instancias de acceso a la docencia a través de un sistema de méritos. ${ }^{41}$

Más allá de estos contrates, el movimiento de la Reforma, en su faceta educativa, actuó como un punto común en la canalización de demandas de renovación de la enseñanza universitaria en distintos aspectos como la docencia libre, la defensa de los concursos para la composición de las ternas docentes, el intercambio científico, etc. Según ha estudiado Buchbinder estas nuevas reglamentaciones, si bien lograron limitar considerablemente el peso de los criterios de origen social o pertenencia política en el acceso a la carrera académica, sustituían esos criterios por otros más afines a la corporación profesional (2010:140). En esa dirección, estas revistas ejercieron una función de vigilancia sobre los mecanismos de reclutamiento dentro del campo profesional-académico. De modo específico, el contraste entre las revistas nos permite plantear algunas particularidades que adoptó ese proceso en las distintas casas de estudio.

En el caso de Córdoba, desde El Bisturí la defensa de los criterios de probidad profesional en los nombramientos de la facultad y la de asistencia pública se materializó en una acusada denuncia contra la injerencia y el favoritismo de la elite médica a cargo de la FCM y del Círculo Medico local. Sin embargo, esta función impugnadora no solamente expresaba una disputa de los nuevos aspirantes por el acceso a los cargos y sus demandas a favor de nuevos mecanismos de legitimación profesional, sino que, como vimos en el apartado anterior, también se combinaba con proyectos que buscaban la renovación de la función científica y social de la universidad como los que gestionaron los líderes reformistas Gregorio Bermann y Enrique Barros. Asimismo, la función crítica de El Bisturí se ejercía recurriendo al contraste con los recursos, mecanismos y disposiciones que se establecían en paralelo en la UBA en aspectos cruciales de la modernización de la enseñanza, lo cual no solo expresaba las aludidas diferencias en los procesos institucionales de reforma de ambas casas de estudio, que en Buenos Aires habían comenzado con la reforma de 1906, sino que también podía aludir a las aspiraciones profesionales de estos grupos de validar y posicionar sus credenciales educativas dentro del sistema educativo que se ampliaba en el nivel superior con la creación de nuevas universidades.

Por el contrario, en las revistas porteñas la defensa de los criterios profesionales en la enseñanza y en la selección del profesorado se referenciaba en las medidas

\footnotetext{
${ }^{41}$ La reforma de 1906 establecía la reglamentación de la docencia libre. Sin embargo, esa disposición solo se llegó a tratar en la FCM, aunque sin aplicación práctica hasta su reformulación con la Reforma de 1918. Pese a ello, sí se llegaron a implementar otras instancias de docencia a partir de la sanción de la carrera de profesor adscripto (Carreño, 2018).
} 


\section{Entre la intervención política y la construcción de un ethos profesional. La red de revistas estudiantiles independientes de medicina en la década del veinte}

sancionadas en la reforma de 1906 y ampliadas en la Reforma de 1918. En La Cureta ello se observa por ejemplo en la promoción del proyecto de docencia libre promovido por el profesor Aráoz Alfaro, quien había sido uno de los principales impulsores del proceso reformista de principios de siglo. ${ }^{42}$ Ese reconocimiento da cuenta de cierto consenso que vinculó a los estudiantes reformistas con un sector del profesorado que, pese a no apoyar el principio reformista del cogobierno, auspiciaban la creación de instancias de investigación y de mejoramiento de la enseñanza (Calderari \& Funes, 1998), en tanto que dicho profesor se había posicionado en contra de la participación de los estudiantes en gobierno de la Facultad. Ese mismo consenso se expresó en la defensa que se hizo desde esa revista, y desde el CMA-CEM, del proyecto, auspiciado por el cuerpo de profesores, de implementación del examen de ingreso en la Facultad. Esta medida enfrentó las posiciones de los estudiantes porteños de medicina y las Federaciones Universitarias de Córdoba (FUC) y del Litoral (FUL), las cuales se manifestaron en contra del proyecto ante la Federación Universitaria Argentina (FUA), agravando el distanciamiento entre esta última organización y la Federación Universitaria de Buenos Aires (FUBA) que defendió la postura del CMA-CEM. ${ }^{43}$

Más allá de estas diferencias, las tres revistas coincidieron en otros aspectos en la constitución de un ideal profesional. Por un lado, un tema que las relacionaba se vincula con la preocupación sobre las condiciones socio-económicas que acompañaban el ejercicio de la profesión, lo cual puede relacionarse con el proceso de diversificación de la matrícula al que aludimos más arriba. Tal como ha señalado Adamovsky, en su análisis sobre las demandas de los profesionales universitarios, en el periodo de entreguerras no se registra que la defensa de los intereses gremiales haya dado lugar al surgimiento de una identidad de "clase media". Según este autor, los primeros pasos del asociacionismo de los diplomados tuvieron que ver más con la necesidad de controlar la administración y el reconocimiento estatal de cada "ciencia" que, con iniciativas mutuales o reclamos de tipo económicogremial, que recién se hicieron camino en la década del 30, aunque según la profesión se reconocen antecedentes previos (2011). En este último sentido, se advierte que las revistas de medicina comenzaron a discutir algunos aspectos económicos-gremiales de la profesión médica que ganarían protagonismo en la próxima década, ${ }^{44}$ cuando se los empezó a percibir como síntomas de una crisis del ejercicio de la práctica liberal de la medicina (Belmartino, Bloch, Persello \& Carnino, 1988: 15-31).

\footnotetext{
42 Salaverry, C. La docencia Libre (25/09/1918). La Cureta, I(5), 1918, p. 101.

43 Bosch, R. Nuestras asociaciones estudiantiles (ab. 1919). Revista del CMA-CEM, XIX (212), pp. 375385.

44 En La Cureta se criticaba las condiciones de explotación en las "Sociedades de Socorros Mutuos" que actuaban como verdaderas empresas capitalistas (La explotación de los profesionales (25/08/1918). La Cureta, I (3), pp. 53-56.), mientras que en El Cocobacilo se satirizaba sobre las dificultades en percibir los honorarios médicos (Dr. Veresaietl, Los honorarios médicos (ab. 1920). El Cocobacilo, II (9) pp. 9-11).
} 


\title{
Luciana Carreño
}

Por otro lado, en relación a lo anterior, un tema predominante aludía a una serie de factores (el curanderismo, la charlatanería, la competencia de productos farmacéuticos sin recetas) que contribuían agravar esas condiciones de ejercicio profesional. Sin embargo, a diferencia de otras críticas periodísticas que recalaban en la inacción y falta de regulación del Estado frente al ejercicio ilegal de la medicina (Rivero, Carbonetti \& Rodríguez, 2017), acorde con el público al que se dirigían, las críticas de estas revistas apuntaban contra la "moral de algunos médicos" y estudiantes que recurrían a estas prácticas dentro de un mercado laboral que se advertía como más competitivo y democrático. ${ }^{45}$ Esta apelación moral también se aplicaba en contra de los estudiantes que recurrían a la adulación, al uso de influencias personales y al dinero como medios de ascenso dentro de la universidad. De este modo, estas críticas condensaban un discurso meritocrático que acusaba sobre el papel que se otorgaba en la universidad al capital social y político en las distintas trayectorias escolares. En contraposición y desde una afirmación del capital académico, la sátira de El Cocobacilo (reproducida posteriormente desde EI Bisturí) apuntaba en contra de los apellidos, las recomendaciones y los parentescos:

\begin{abstract}
"Con motivo del éxito obtenido en su cuarto examen de histología. Los admiradores de Saturniano Candelejas Unzué han resuelto obsequiarle una medalla de oro. La medalla tiene... la siguiente inscripción: "con paciencia y lo demás se tiene éxito".46

- ¿Es Ud. pariente de fulano? ...Cada cual es hijo de sus propias obras... y no asiste ningún derecho al examinador inquirir antecedentes de familia por cuanto estos no pueden ni deben influir en su ánimo. ¡Es justicia señores examinadores adulones! ${ }^{47}$
\end{abstract}

Al mismo tiempo, el caso de El Cocobacilo y El Bisturí no solo es ilustrativo de la legitimación por medio del capital escolar que hacían estos sectores sociales en ascenso, sino también de un proceso de emancipación de los judíos (Hobsbawm, 2013:69-82), que se realizaba en este caso a través de las credenciales educativas en instituciones estatales- laicas y la participación en distintas asociaciones. ${ }^{48}$ Finalmente, la función moral que se advierte en estas críticas también apuntó a discutir y asentar mecanismos de solidaridad gremial dentro del estudiantado en demandas relativas a la práctica profesional. A tono con otros emprendimientos gremiales que se replicaban en otras ciudades del país (Adamovsky, 2011), las páginas de El Bisturí dan cuenta de la discusión de iniciativas a favor de la creación de un sindicalismo universitario. Para ello la revista ironizaba sobre los excesivos temores que despertaba ese proyecto, que retomaba los modos de organización del movimiento obrero, en ambiente cordobés como “expresión neta y nata del

\footnotetext{
45 Dolhare, F. La moral de algunos médicos (dic. 1924). El Bisturí, II (24), pp. 14-15.

46 Sociales (oct. 1920). El Cocobacilo, II (15), p. 8.

${ }^{47} \mathrm{Al}$ margen de los exámenes. ¿Es Ud. pariente de fulano? (dic. 1920). El Cocobacilo, II (17), p. 11.

48 Sobre ese proceso de emancipación en relación a la profesión médica véanse: Los Médicos en la

Edad Media y Del Tamud. El médico bueno y liberal (enero 1922). El Cocobacilo, IV (29), pp. 3-6 y 11.
} 


\section{Entre la intervención política y la construcción de un ethos profesional. La red de revistas estudiantiles independientes de medicina en la década del veinte}

orden". ${ }^{49}$ Al mismo tiempo, la revista saludaba la medida de la huelga de los practicantes de los hospitales de Buenos Aires, en oposición a la resolución de la Asistencia Pública municipal de suprimir la institución del internado médico. Sin embargo, la fallida realización de esa huelga, expresa las tensiones dentro del estudiantado (que repercutieron incluso dentro de la misma red de revistas) ${ }^{50}$ y las limitaciones que enfrentaban estas publicaciones en su función de socializar y compatibilizar nuevos principios reformistas de intervención pública y de ética para el ejercicio profesional.

\section{Conclusiones}

El enfoque adoptado en este estudio apuntó a desarrollar una aproximación a las revistas universitarias, en la que se rescataron las complejas articulaciones entre las formas de intervención política-intelectual propias del reformismo y otras cuestiones centrales de la vida universitaria y de la proyección profesional de los estudiantes que participaban en estas iniciativas editoriales. El análisis de los procesos de diversificación de la matrícula; de profesionalización de las carreras y disciplinas universitarias y de democratización y organización de las entidades estudiantiles nos permitió contextualizar la emergencia de la red de publicaciones independientes de medicina y advertir una serie de tensiones y particularidades en la construcción de dichas interconexiones entre los ideales de la Reforma y aquellos propios de la profesionalización.

Por un lado, esa conjunción se advierte en la recuperación de ciertas modalidades de intervención pública que formaban parte de las estrategias de legitimación social de la corporación médica desde finales del siglo XIX, de la mano del higienismo y de la figura del médico social. Sin embargo, el contexto ideológico marcado por el movimiento reformista y por el impacto de la Gran Guerra y la Revolución rusa, implicó una reformulación de estas prácticas a través de la realización de la extensión universitaria y de una función crítica de las injusticias de un orden social en el que se limitaban las posibilidades de acción los profesionales. Asimismo, según señalamos en el caso cordobés, la vertiente profesional también podía conjugarse en estas revistas con el diseño de proyectos que aspiraban a desarrollar la función social de la universidad, de la mano del intercambio universitario, la reforma de los planes de estudios y la creación de institutos.

Por otro lado, los diferentes procesos de profesionalización en cada universidad, permiten considerar las distintas modalidades que adquirían esos intereses en su interrelación con la universidad. Así, en el caso cordobés, la función impugnadora

49 El Cuco (jul. 1923). El Bisturí. I (4), pp. 6-7.

50 El Bisturí celebraba también la medida del CMA-CEM de publicar en su revista una lista de "traidores y perjuros" que rompieron la huelga al aceptar puestos en la asistencia pública. Dentro de la lista figuró el nombre del José Svibel, quien actuó por un tiempo como director de El Cocobacilo. 


\section{Luciana Carreño}

que ejerció EI Bisturí contribuía a suplir y contrastar la función de complementariedad que asumió el asociacionismo corporativo del Círculo Médico en relación con la FM. Por el contrario, el asociacionismo estudiantil porteño no sólo resultaba heredero de las entidades médicas que, en el siglo XIX, se opusieron a la elite que controlaba la facultad y que contribuyeron a instaurar mecanismos de reclutamiento abierto al juicio de pares, sino que también actuó como una pieza central para movilizar el desarrollo de una reforma institucional desde 1906. Esas diferencias ayudan a comprender las particularidades y tensiones en el campo estudiantil, en cual, por ejemplo, se cita el conflicto que enfrentó a los estudiantes porteños con sus pares de Córdoba y el Litoral, por la aceptación de los primeros del proyecto de implementación de los exámenes de ingreso.

Otro punto de contacto, en el cual se articulaba la conexión entre el reformismo y la construcción de un ideal profesional, puede vincularse con el proceso de diversificación de la matrícula que se operaba, especialmente en la universidad porteña, desde principios de siglo. En ese aspecto, el componente inmigratorio e ideológico que observamos en estas revistas se expresó a través de la crítica satírica y desde una marcada apelación moral que aspiraba socializar al mismo tiempo nuevas conductas "de conciencia progresista" entre los estudiantes (a tono con ciertos postulados del reformismo en su vertiente extra-institucional) y nuevos preceptos de ética profesional que comenzaban desde de la misma formación universitaria. En este sentido, la prédica democratizadora que se observaba en las críticas a los "niños bien" (en la condena a la movilización del capital social en los exámenes, la obsecuencia o la falta de rigurosidad en los estudios) se emparentaba con las críticas hacia los egresados que acusaban de "charlatanismo médico" o de prescribir de medicamentos indiscriminadamente en acuerdo con las droguerías, que minaban el prestigio y las condiciones de ejercicio de la vida profesional.

Finalmente, la exclusión de las mujeres (quieres también formaban parte, por cuestiones de género, del proceso de diversificación de la matrícula) por fuera de esta prédica meritocrática del ideal profesional, señala otro de los posibles temas de estudio a profundizar en el análisis de las articulaciones entre reformismo, profesionalización y cambios sociales en la universidad.

\section{Bibliografía}

Adamovsky, E. (2011). Gremialismo y política en los profesionales universitarios: el problema de la identidad de clase durante la primera mitad del siglo XX. Redes, 17 (33), pp. 35-61.

Agüero, A. C. (2017). Local/nacional: una historia cultural de Córdoba en el contacto con Buenos Aires (1880-1918). Bernal: Universidad Nacional de Quilmes Editorial.

Bargero, M. (2002). Condiciones institucionales y culturales de la enseñanza de la 


\section{Entre la intervención política y la construcción de un ethos profesional. La red de revistas estudiantiles independientes de medicina en la década del veinte}

medicina en Buenos Aires: reformas académicas y movimientos estudiantiles entre 1874 y 1906. Entrepasados, (22), pp. 91-112.

Belmartino, S., Bloch, C., Persello, A. V. \& Carnino, M. I. (1988). Corporación médica y poder en salud. Argentina, 1920- 1945. Rosario: Centro de Estudios Sanitarios y Sociales.

Biagini, H. (2012). La contracultura juvenil. De la emancipación a los indignados. Buenos Aires: Capital Cultural.

Buchbinder. P. (2010). Historia de las Universidades Argentinas. Buenos Aires: Sudamericana.

Bustelo, N. (2015). La reforma universitaria desde sus grupos y revistas. Una reconstrucción de los proyectos y las disputas del movimiento estudiantil porteño de las primeras décadas del siglo XX (1914- 1928). (Tesis de doctorado inédita). Universidad Nacional de La Plata, La Plata.

Bustelo, N. \& Domínguez Rubio, L. (2017). Radicalizar la Reforma Universitaria. La fracción revolucionaria del movimiento estudiantil argentino, 1918-1922. Anuario Colombiano de Historia Social y de la Cultura, 44 (2), pp. 31-62.

Calderari, M. \& Funes, P. (1998). Escenas reformistas. La Reforma Universitaria 1918-1930. Buenos Aires: Eudeba.

Carreño L. (2018). Reformismo y renovación educativa. Trayectorias universitarias y participación estudiantil en torno a la docencia libre en la Facultad de Ciencias Médicas de la Universidad de Buenos Aires. De signos y sentidos, (19), pp. 51-77.

Carreño, L. (2020). Los estudiantes universitarios en tiempos de reformas. Sociabilidad y vida estudiantil en la universidad porteña (1900-1930). Buenos Aires: Eudeba [en prensa].

Celentano, A. (2014). Por la ciencia y la Reforma Universitaria: las revistas de los estudiantes de Medicina de La Plata (1921-1925). Los Trabajos y los Días. VI (4-5), pp. 194- 213.

Di Liscia, M. S. (2002). Saberes, Terapias y Prácticas Médicas en Argentina (17501910). Madrid: Consejo Superior de Investigaciones Científicas.

Frederic, S., Graciano O. \& Soprano, G. (2010) (coord.). El Estado argentino y las profesiones liberales, académicas y armadas. Rosario: Prohistoria Ediciones.

Garzón Maceda, F. (1916). La medicina en Córdoba. Apuntes para su historia. Tomo I. Buenos Aires: Talleres Gráficos Rodríguez Giles. 


\section{Luciana Carreño}

González Leandri, R. (1999a) Curar, persuadir y gobernar. La construcción histórica de la profesión médica en Buenos Aires, 1852-1886. Madrid: Consejo Superior de Investigaciones Científicas.

González Leandri R. (1999b). Las profesiones. Entre la vocación y el interés corporativo. Fundamentos para su estudio histórico. Madrid: Catriel.

Graciano, 0. (2008). Entre la torre de marfil y el compromiso político. Intelectuales de izquierda en la Argentina, 1918- 1955. Bernal: Universidad Nacional de Quilmes.

Graciano, 0. (2016). Pensar la sociedad, intervenir en política: la universidad argentina y sus estrategias de participación en la vida pública y en la política nacional, 1918-1945. Revista IRICE, (31), pp. 159-193.

Hobsbawm, E. (2012). Un tiempo de rupturas. Sociedad y cultura en el siglo XX. Barcelona: Crítica.

Ingenieros, J. (1920). La Universidad del Porvenir. Ateneo (3). Buenos Aires: Publicaciones del CMA-CEM.

Lorenzo, F. (2016). “Que sepa coser, que se pasa bordar, que sepa abrir para la puerta para ir a la Universidad". Las académicas en la Universidad de Buenos Aires en la primera mitad del siglo XX. Buenos Aires: Eudeba.

Ministerio de Justicia e Instrucción Pública (1926). Estadística. (Anexo a la Memoria del Ministerio). 1925. Buenos Aires: Talleres Gráficos de la Penitenciaría Nacional.

Pita González, A. (2009). La Unión Latinoamericana y el Boletín de Renovación: Redes de intelectuales y revistas culturales en la década de 1920. Ciudad de México: El Colegio de México, Universidad de Colima.

Rivero, M. D. (2019). Dinámicas publicitarias en materia de aparatología terapéutica y farmacología: una aproximación a los mercados de Córdoba, Rosario y Buenos Aires (1912-1938). (Tesis de doctorado). Universidad Nacional de La Plata, La Plata.

Rivero, M. D. Carbonetti, A. \& Rodríguez, M. L. (2017). Alternativas al saber diplomado en la escena pública: una aproximación al curanderismo a partir de la prensa escrita de las ciudades de Córdoba y de Buenos Aires, Argentina en la década de 1920. Historia y Sociedad, (33), pp. 19-43.

Rodríguez, F. (1999). Inicial, Sagitario y Valoraciones. Una aproximación a las letras y a la política de la Nueva generación americana. En S. Sosnowaski (ed.) La Cultura de un siglo: América Latina y sus revistas. Buenos Aires: Alianza.

Rodríguez, M. L. (2007). La elite médica y su definición interna como grupo de poder en Córdoba, 1878 y 1923. Ponencia presentada en XI Jornadas Interescuelas, San 
Entre la intervención política y la construcción de un ethos profesional. La red de revistas estudiantiles independientes de medicina en la década del veinte

Miguel de Tucumán, Tucumán.

Sarfatti Larson M. (1989). Acerca de los expertos y las profesiones o la imposibilidad de haberlo dicho todo. Revista de Educación (Supl. 1), pp. 199-225.

Schellemberg, A. (1982). Del Mar Negro al Río de La Plata. Buenos Aires: [el autor].

Schenone, G. (2009). La protesta universitaria de Córdoba en 1924. Su filiación con la Reforma de 1918. Cuadernos de Historia, (11), pp. 163-186.

Tarcus, H. (2007). Diccionario biográfico de la izquierda argentina. Buenos Aires: Emecé.

Vagliente, P. (2016). Asociativa, movilizada, violenta. La vida pública en Córdoba, 1850- 1930. Tomos I y II. Villa María: Eduvim.

Vallejo, G. (2018). La hora cero de la eugenesia en la Argentina: disputas e ideologías en el surgimiento de un campo científico, 1916-1932. História, Ciências, Saúde Manguinhos, (25), pp.15-32.

Vezzetti, H. (1985). La locura en Argentina. Buenos Aires: Paidós.

Recibido: 30/04/2020

Evaluado: $15 / 06 / 2020$

Versión Final: 29/07/2020 